Keller, Andreas:

\title{
20 Jahre Strahlenschutzausbildung an der TH/TU IImenau
}

\author{
Zuerst erschienen in: Biomedizinische Technik = Biomedical Engineering. - Berlin [u.a.] : \\ de Gruyter. - 46 (2001), S1, S. 456-457. \\ Jahrestagung der Deutschen Gesellschaft für Biomedizinische \\ Technik (DGBM) im VDE ; 35 (Bochum) : 2001.09.19-21 \\ Erstveröffentlichung: 2001 \\ Datum Digitalisierung: $2009-08-06$ \\ ISSN (online): $\quad$ 1862-278X \\ ISSN(print) 0013-5585 \\ DOI: $\quad$ 10.1515/bmte.2001.46.s1.456 \\ [Zuletzt gesehen: 2019-12-06]
}

„Im Rahmen der hochschulweiten Open-Access-Strategie für die Zweitveröffentlichung identifiziert durch die Universitätsbibliothek IImenau."

"Within the academic Open Access Strategy identified for deposition by IImenau University Library."

„Dieser Beitrag ist mit Zustimmung des Rechteinhabers aufgrund einer (DFGgeförderten) Allianz- bzw. Nationallizenz frei zugänglich."

"This publication is with permission of the rights owner freely accessible due to an Alliance licence and a national licence (funded by the DFG, German

Research Foundation) respectively."

\section{DFG}

Nationallizenzen 


\title{
20 JAHRE STRAILENSCHUTZAUSBILDUNG AN DER TH/TU ILMENAU
}

\author{
A. Keller \\ Institut f. Biomedizinische Technik und Informatik, Techn. Univ. IImenau, Deutschland \\ andreas.keller@informatik.tu-ilmenau.de
}

\section{I:INI.IIITUNG}

Scit Gründung des Institutes für Elektromedizinische und Radiologische Technik im Jahre 1953 an der damaligen Hochschule für Elektrotechnik in IImenau ist eine Integration von Strahlenphysik und Strahlentechnik in Ausbildung und Forschung der Biomedizinischen Technik erfolgt. Über alle Hochschulreformen hinweg und auch in den Umstrukturierungen der Wende und nach der deutschen Wiedervereinigung hat sich dieses Konzept bewährt und erhalten. Mit einem umfangreichen Lehrgebietskomplex ionisierender Strahlen in der Medizin im Hintergrund entstand bereits 1979 die Idee, auf dieser vorhandenen Basis eine staatlich anerkannte Strahlenschutzausbildung in das Studium zu integrieren.

\section{AUSBILDUNG BIS 1990}

Mit insgesamt 195 Stunden zu den Themen Strahlenphysik, Strahlenbiologie, Anatomie, Physiologie, Klinische Verfahren (einschließlich Klinischer Radiologic), Radiologische Technik, Strahlungsindikatoren und Strahlungsmessgeräte sowic Dosimetrie energiereicher Strahlung war ein Umfeld im Studium gegeben, welches eine hinreichende Grundlage für eine Strahlenschutzausbildung zum Erwerb des damaligen Staatlichen Befähigungsnachweises für Strahlenschutzfachkräfte darstellte.

Im Mai 1981 schloss hierzu die Technische Hochschule Ilmenau eine Vereinbarung mit dem Staatlichen Amt für Atomsicherheit und Strahlenschutz Berlin (SAAS) ab. Das SAAS hatte in der DDR das Ausbildungsmonopol zum Erwerb von Fachkenntnissen im Strahlenschutz. Deshalb musste im Vertrag geregelt werden, dass abweichend hiervon das SAAS Lehrleistungen anderer Einrichtungen akzeptierte. In Anerkennung der Ausbildungsinhalte fand für die Ilmenauer Studenten am Ende des Studiums nach bestandener Hauptprüfung ein verkürzter Ilmenauer Sonderkurs jeweils zur Hälfte in Ilmenau und in Berlin statt. Bei erfolgreichem Kenntnisnachweis wurde für die Kursteilnehmer der Staatliche Befähigungsnachweis für Strahlenschutzfachkräfte für das Arbeitsgebiet "Anwendung ionisierender Strahlung in der Medizin" erteilt. Dies umfasste also alle Disziplinen der Klinischen Radiologie, in denen naturwissenschaftlich-technische Hochschulabsolventen eingesetzt werden konnten: Röntgendiagnostik, Nuklcarmedizin und Strahlenthera- pie. Seit 1980 waren die Sonderkurse Bestandteil des Studienplanes und gemeinsam mit dem Diplom erwarben die Absolventen den Staatlichen Befähigungsnachweis für Strahlenschutzfachkräfte.

\section{AUSBILDUNG ZWISCHEN 1990 UND 1992}

Mit dem Staatsvertrag zur Wirtschafts-, Währungsund Sozialunion vom 18. Mai 1990 galt das Umweltrecht der BRD in der DDR und damit auch Strahlenschutz- und Röntgenverordnung. Dies machte die Umstellung der Ausbildung mitten im Semester erforderlich. Das gelang auch mit personeller Unterstützung aus den alten Bundesländern von der FH Giessen-Friedberg. Vorübergehend anerkannten die Behörden nur den Abschluss für das Spezialgebiet Röntgendiagnostik. Aber gerade hier liegt kein typischer Einsatzfall Ilmenauer Absolventen als Strahlenschutzbeauftragte für den physikalisch-technischen Bereich. Um einen breiten und arbeitsmarktwirksamen Abschluss in das Studium zu integrieren, mussten sowohl die Zugangsvoraussetzungen als auch der Sonderkurs für unsere Absolventen grundlegend überarbeitet werden. Das Vorhaben orientierte sich an den Erfordernissen der einschlägigen Fachkunderichtlinien als Mindestanforderungen.

\section{AUSBILDUNG SEIT 1993}

Über eine Reihe von Zwischenlösungen hat jetzt die Strahlenschutzausbildung als Teil des Erwerbs der Fachkunde folgende Gestalt: Im Regelstudium (Studiengang Elektrotechnik, Studienrichtung Biomedizinische Technik und Informatik) werden mit einem Umfang von insgesamt 225 Stunden in didaktisch optimaler Reihenfolge die Lehrgebiete in Tabelle 1 angeboten:

Tab. 1: Zugangsvoraussetzungen des Regelstudiums

\begin{tabular}{|l|c|c|}
\hline Lehrgebiet & Semester & Stunden \\
\hline Anatomie, Physiologie & 5. & 30 \\
\hline Strahlenbiologie & 5. & 15 \\
\hline Strahlenphysik & 5. & 15 \\
\hline Klinische Verfahren & 6. & 30 \\
\hline Strahlungsmesstechnik & 6. & 30 \\
\hline Bildgebende Systeme & 8. & 45 \\
\hline Klinische Dosimetrie & 9. & 30 \\
\hline Strahlenschutz & 9. & 30 \\
\hline
\end{tabular}


Da bei der Integration einer solchen Ausbildung in cin Regelstudium die nicht vorhandene Praxiserfahrung Grenzen setzt, welche wenigstens teilweise zu kompensieren sind, sind fakultative Spezialseminare über 45 Stunden im Angebot und den Zugangsvoraussetzungen zugeordnet. Diese Spezialseminare werden in Kooperation mit klinischen Einrichtungen und von praxiserfahrenen Gastreferenten durchgeführt. Allein in das Spezialseminar Bildgebende Diagnostik sind 5 Kooperationseinrichtungen mit 13 Gastreferenten integriert.

In Ilmenau selbst stehen ein Röntgenstrahlen- und ein Kernstrahlenlabor für Praktika zur Verfügung. Das Röntgenstrahlenlabor ist mit einem Konvertergenerator und zwei Anwendungsgeräten (Decken-Boden-Stativ mit Rasteraufnahmetisch und Rasterwandstativ, Kipptisch mit Röntgenbildverstärker und hochauflösender Röntgenfernsehkette) ausgestattet. Zur experimentellen Veranschaulichung der Querschnittsrekonstruktionsverfahren wurde ein experimenteller Computertomograph selbst entwickelt. Dosis- und Dosisleistungsmessung sind gleichzeitig mit 6 Messgeräten möglich. Das Kernstrahlenlabor ist ausgerüstet für Arbeiten mit offenen und umschlossenen Radionukliden. Neben einer Grundausstattung mit Strahlungsmesstechnik und Strahlenschutzmesstechnik stehen u.a. ein stickstoffgekühlter Reinstgermanium-Detektor zur Gammaspektrometrie und eine Szintillationskamera zur Bildgebung zur Verfugung. Diese umfangreichen experimentellen Voraussetzungen werden in Praktika des Regelstudiums als auch in Praktika des llmenauer Sonderkurses genutzt.

Im 10. Semester findet als Blockveranstaltungen der Sonderkurs mit einem Umfang von 45 Stunden statt. Das didaktische Konzept nutzt den Risikobegriff als Gliederungskriterium (Tab. 2). $\mathrm{Zu}$ den Spezialgebieten werden die relevanten Themen jeweils spezifiziert.

Tab. 2: Didaktisches Konzept der Ausbildung

\begin{tabular}{|c|c|}
\hline Grundlagen & Themen \\
\hline Risikoursache: & - Strahlenanwendung \\
\hline Risikoqualităt: & $\begin{array}{l}\text { - Strahlenphysik } \\
\text { - Strahlenbiologie } \\
\text { - Strahlenrisiko }\end{array}$ \\
\hline Risikoquantităt: & $\begin{array}{l}\text { - Strahlungsmesstechnik } \\
\text { - Strahlenschutzdosimtrie } \\
\text { - Exposition des Menschen }\end{array}$ \\
\hline Risikoakzeptanz: & - Grundsätze \\
\hline Risikoumgang: & $\begin{array}{l}\text { - Recht } \\
\text { - behördliche Verfahren } \\
\text { - Überwachung, Kontrolic } \\
\text { - Strahlenschutztechnik } \\
\end{array}$ \\
\hline Schaden: & - Stör und Unfalle \\
\hline Spezialgebiete & Themen \\
\hline $\begin{array}{r}\text { Rontgendiagnostik } \\
\text { Nuklearmedizin } \\
\text { Strahlentherapic } \\
\text { Techn. Anwendgn. }\end{array}$ & $\left\{\begin{array}{l}\text { Dosimetrie } \\
\text { Recht, hehordliche Verfahren } \\
\text { Oberwachung und Kotrolle } \\
\text { Strahlenschutztechnik } \\
\text { Stor- und Unfalle }\end{array}\right.$ \\
\hline
\end{tabular}

\section{TRÄGERSCHAFT UND KOOPERATIONEN}

Um Röntgen- und Kernstrahlenlabor mit ihren medizinischen Bilderzeugungssystemen auch ohne jeden Patientenbetrieb auf Dauer unterhalten zu können, wurde 1994 das Strahlenschutzseminar in Thüringen als eingetragener, gemeinnütziger und besonders förderwürdiger Verein gegründet. Satzungsgemäß fördert er mit Sach- und Geldmitteln Erhaltung und Modemisierung beider Labore. Da der Verein bundesweit anerkannter Kursanbieter ist, übernahm er in Kooperation mit der TU Ilmenau auch die Trägerschaft für den Ilmenauer Sonderkurs. Das Strahlenschutzseminar sichert die personellen und materiellen Voraussetzungen für den Kurs. Die TU Ilmenau sichert die Zugangsvoraussetzungen im Studium, auf denen der Sonderkurs aufbaut. Die Kursteilnahme ist für die Studenten kostenfrei.

Die zuständige Behörde, das Thüringer Ministerium für Landwirtschaft, Naturschutz und Umwelt, bestätigte alle Voraussetzungen für eine entsprechende Anerkennung dieses Abschlusses zu insgesamt 15 Anwendungsgebieten bzw. Tätigkeitsgruppen nach vier Fachkunderichtlinien. Hierzu gehört auch der Strahlenschutzkurs für Medizinphysiker und "sonstige hinreichend ausgebildete Personen", die als Strahlenschutzbeauftragte für physikalisch-technische Aufgaben in der Strahlentherapie und Nuklearmedizin bestellt werden sollen.

Weitere Kooperationspartner sind das Zentralklinikum Bad Berka GmbH (Institut für Bildgebende Diagnostik und PET-Zentrum), das Zentralklinikum Suhl gGmbH (Klinik für Nuklearmedizin), das Klinikum Erfurt GmbH (Klinik für Strahlentherapie), SIEMENS Medizinische Technik Erlangen und Forchheim, SIEMENS Rőntgenwerk Rudolstadt, die Radiologische Praxis Frau Dr. Eger Rudolstadt und das Institut für Werkstoffe der TU llmenau. Mit technischen Ausrüstungen unterstützen die Strahlenschutzausbildung u.a. PTW Freiburg, Hofmann Dornier Medizintechnik Erlangen, Agfa Leverkusen sowie die Sensortechnik und Elektronik GmbH Pockau.

In den vergangenen 20 Jahren wurden insgesamt 451 Absolventen mit einer Strahlenschutzausbildung in dic Praxis entlassen und die Erfahrungen vor allem der letzten Jahre zeigen, dass der bestätigte und bundesweit anerkannte Kursbesuch auf dem Arbeitsmarkt wirhsam wird. Bei allen Grenzen der fehlenden Praxiserfahrung stellt diese Ausbildung ein Beispicl dar für eine wirksame Effektivierung der universitären Ausbildung durch Integration von postgradualen Ausbildungen in das Studium.

apl. Prof. Dr.-Ing. habil. Andreas Keller

Institut für Biomedizinische Technik und Informatik

Technische Universităt llmenaau $\mathrm{Pl}: 100565$

1). 98684 llimenau 